\title{
The Comparison of Antimicrobial Effect of Nigella sativa Nanoparticle and Chlorhexidine Emulsion on the Most Common Dental Cariogenicic Bacteria
}

\author{
Bahareh Nazemi Salman ${ }^{1}$, Shilan Sallah ${ }^{2}$, Fatemeh Abdi ${ }^{3}$, Sarvenaz Salahi ${ }^{4}$, Kobra Rostamizadeh $^{5}$, Samira Basir \\ Shabestari ${ }^{6} *$ (D)
}

Received: 14 Feb 2021

Published: 8 Nov 2021

\begin{abstract}
Background: Tooth decay and periodontal disease are the most common chronic human and oral diseases, respectively, and bacterial plaque has a major role in their occurrence. Because of the importance of plaque control, this study was done to compare the antimicrobial effects of Nigella sativa nanoparticles and chlorhexidine emulsion on the most common dental cariogenicic bacteria.

Methods: In this experimental study, the effects of $0.2 \%$ chlorhexidine mouthwash and Nigella sativa nanoparticle with different dilutions on Streptococcus mutans, Streptococcus sobrinus, Streptococcus salivarius, Lactobacillus acidophilus, Minococcal fecalis, and Enterococcus fecalis were compared using minimum inhibitory concentration (MIC) and minimum bactericidal concentration (MBC) assessment. Data were analyzed by SPSS Version 16.0 software, and statistical tests, including an independent sample $t$ test.

Results: Mean diameters of growth inhibition zone because of the nanoemulsion of Nigella sativa nanoparticle was close to each other in different bacteria $(\mathrm{p}=0.665)$. In addition, there was no significant difference between these values because of different dilutions of nanoemulsion even in different microbial species $(\mathrm{p}=0.778)$. The MIC and lethal concentrations of Nigella sativa nanoemulsion were similar for Enterococcus faecalis and Streptococcus mutans, and it was higher than other bacteria. In comparison, the MIC and MBC values of all bacteria in chlorhexidine were lower than those of the nanoemulsion.

Conclusion: MIC and MBC values showed that Nigella sativa nanoemulsion affects tooth cariogenicic bacteria. Enterococcus faecalis and Lactobacillus acidophilus were the most resistant and susceptible bacteria to this nanoparticle, respectively, while the antimicrobial effects of Nigella sativa nanoemulsion were weaker than the chlorhexidine mouthwash.
\end{abstract}

Keywords: Nigella sativa, Nanoemulsion, Chlorhexidine, Dental Caries, Bacteria, Antimicrobial

Conflicts of Interest: None declared

Funding: None

*This work has been published under CC BY-NC-SA 1.0 license.

Copyright $\odot$ Iran University of Medical Sciences

Cite this article as: Nazemi Salman B, Sallah Sh, Abdi F, Salahi S, Rostamizadeh K, Basir Shabestari S. The Comparison of Antimicrobial Effect of Nigella sativa Nanoparticle and Chlorhexidine Emulsion on the Most Common Dental Cariogenicic Bacteria. Med J Islam Repub Iran. 2021 (8 Nov);35:149. https://doi.org/10.47176/mjiri.35.149

\section{Introduction}

The mouth is one of the essential organs of the human body that plays a vital role in the nutrition, speech, and beauty of people, thus, its health affects the quality of life

Corresponding author: Dr Samira Basir Shabestari, shabestari.s@iums.ac.ir

1. Department of Pediatric Dentistry, School of Dentistry, Zanjan University of Medical Sciences, Zanjan, Iran

2. Department of Oral Medicine, School of Dentistry, Zanjan University of Medical Sciences, Zanjan, Iran

3. Zanjan University of Medical Sciences, Zanjan, Iran

4. Minimally Invasive Surgery Research Center, Iran University of Medical Sciences, Tehran, Iran

5anjan Pharmaceutical Nanotechnology Research Center, Zanjan University of Medical Sciences, Zanjan, Iran

6. Department of Oral Medicine, School of Dentistry, Firoozgar Clinical Research Development Center, Iran University of Medical Sciences, Tehran, Iran
$(1,2)$. Many syste mic disorders are related to periodontitis and oral diseases. Therefore, the World Health Organization (WHO) has made oral health a part of public health

$\uparrow$ What is "already known" in this topic:

Based on the literature review, a broad spectrum of animal studies has demonstrated the antimicrobial effect of Nigella sativa nanoparticles in one or a limited number of bacteria. Moreover, its antimicrobial mechanism is vague compared with the gold standard plaque controlling agent (Chlorhexidine).

\section{$\rightarrow$ What this article adds:}

For the first time, the effects of different concentrations of Nigella sativa nanoparticles were evaluated on the most common cariogenicic bacteria and compared with chlorhexidine. Its antimicrobial effects were weaker than chlorhexidine. 
(3). Tooth decay is the most common chronic human periodontal disorder and the leading cause of tooth loss. The oral cavity has a complex and diverse variety of microorganisms. Various types of bacteria, including Streptococcus (mutans, subrinus, salivarius, and sanguis), periodontal pathogens (Porphyromonas gingivalis and Actinobacterium ectinobacterium), and Enterococcus faecalis, have been discovered that are responsible for tooth decay through destruction of dental structures $(4,5)$. In this regard, the WHO has approved proper oral hygiene and microbiome control as an essential strategy for preventing these pathological conditions (6).

The most successful plaque control program combines 2 mechanical and chemical removal strategies (7). Chlorhexidine ( $\mathrm{CHX}$ ) has been recognized as a gold standard plaque control agent because of its broad spectrum of antimicrobial properties, stability, and relative toxicity, but its use has recently been limited (8). Concurrent with the expansion of CHX use, several side effects (alteration of bacterial microflora, tooth and soft tissue immunoreactivity, cytotoxicity, and alteration of taste, erosive oral mucosa, and parotid salivary gland swelling) have also been reported (9). Besides, excessive use of antiseptic agents resulted in microbial resistance (10). Therefore, the worldwide need for new accessible, affordable, and pathogen-sensitive antimicrobials with maximum efficacy and minimal side effects is increasing. In this regard, it is possible to achieve the desired results by utilizing modern technology and nonchemical (herbal) compounds. Among these, the black cumin (also named as Nigella sativa or alhabba al-sawdaa or senouj), which belongs to the family of Ranunculaceae, has been recognized for its effective therapeutic effects (11). This plant grows in many countries, including the Mediterranean, North African, and East Asian countries, and thus it has been termed as an herbal miracle of the century by scientific evidence (12). The tiny grains of this plant are rich in bioactive compounds, such as protein, oil, amino acids, vitamins, and unsaturated fatty acids (13). These compounds have multiple therapeutic properties, including antibacterial and antiviral, anti-inflammatory, analgesic, histamine release inhibitor, antihypertensive, antioxidant, hypoglycemic, and liver protective $(14,15)$. Additionally, these compounds have low toxicity. One of the components of Nigella sativa is thymoquinone. In MIC determination, thymoquinone is active against all the strains studied (16). The main antibacterial activity is seen against Streptococcus constellatus with MIC $4 \mu \mathrm{g} / \mathrm{mL}$. The essential oil also shows the strongest activity against Streptococcus mitis, Streptococcus mutans, Streptococcus constellatus, and Gemella haemolysans with MIC $2.13 \mathrm{mg} / \mathrm{mL}$ (17).

Because of the antimicrobial and anti-inflammatory activity of Nigella sativa, researchers believe in the anticaries effects of this compound on dental caries and periodontal diseases. In this regard, animal and laboratory studies in this field were performed by the usual forms to lower latent bacterial count after drinking Nigella sativacontaining water $(18,19)$. Another study showed more antimicrobial activity of Nigella sativa on Streptococcus sanguis than Streptococcus mutans (20). Some studies showed that silver nanoparticles containing these herbal compounds had more potent antimicrobial effects on Streptococcus mutans than gold and zinc nanoparticles (21). It can be used with less tissue toxicity using proper nanocarriers. Therefore, the development and introduction of nano drugs have been of interest to researchers because of their benefits, such as more extended durability, targeted tissue distribution, and reduced side effects.

Considering the importance of plaque controlling in oral health, the adverse effects of chemicals, the antimicrobial activity of nanoparticles, and also the vague antimicrobial mechanisms of Nigella sativa $(11,12)$, we aimed to compare the antimicrobial effect of nanoemulsion of Nigella Sativa and chlorhexidine on common dental cariogenic bacteria, taking a step towards improving the general health of the population.

\section{Methods \\ Collection of Bacterial Isolates}

In this experimental study, the antimicrobial effect of Nigella sativa emulsion (Zanjan region) on common cariogenicic microbial strains was assessed, which included1683 (ATCC 35668) Streptococcus mutans, 1601(ATTC 27607) Streptococcus sobrinus, (ATCC 19258) Streptococcus salivarius, 1643(DSM 20079) Lactobacillus acidophilus, and 1237(NCTC 8213) Enterococcus faecalis. This study was approved by the National Center for Genetic and Biological Resources of Iran and the resources were prepared in lyophilized fashion (Freeze drying, a low temperature dehydration process for the long-term preservation of cells).

\section{Preparation of Nanoemulsion Oil}

After approval of the University Ethics Committee (code: ZUMS.REC.1395.195) and following the environmental principles of nanoemullsion, the Nigella sativa oil was prepared by solvent evaporation. In this study, fennel flower nanomulsion was prepared via the emulsion method. The size of nanoemulsions was determined by the dynamic light scattering (DLS) method. Figures 1 and 2 depict the typical intensity-based and volume-based results of the DLS analysis. Clearly, the symmetric size distribution profile of nanoemulsions in terms of intensity-based and volume-based particle size distribution as well as low polydispersity index indicates very homogenous properties of nanoemulsion. The $\mathrm{z}$ average of the nanoparticles size was $76.26 \pm 0.15 \mathrm{~nm}$, with the corresponding poly dispersity index of 0.277 . The surface charge of nanoparticles plays a crucial rule in the stability of nanoemulsion. The zeta potential of nanoparticles was determined to be $29.6 \mathrm{mV}$ (Fig. 3). It is believed that owning surface charge over $\pm 20 \mathrm{mV}$ could provide suitable electrostatic repulsion characteristic to make nanoparticles stable for a long time. Considering the high zeta potential of the nanoparticles, it seems that the nanoemulsion would possess a long-term stability. The structure of nanoemulsions consists of nanocrystalline liquid cells, which were dispersed in another liquid that we first weighed a certain amount of Nigella sativa (Zanjan spp.), dissolved it in a suitable solvent (ethanol), and added and dissolved the deionized water con- 


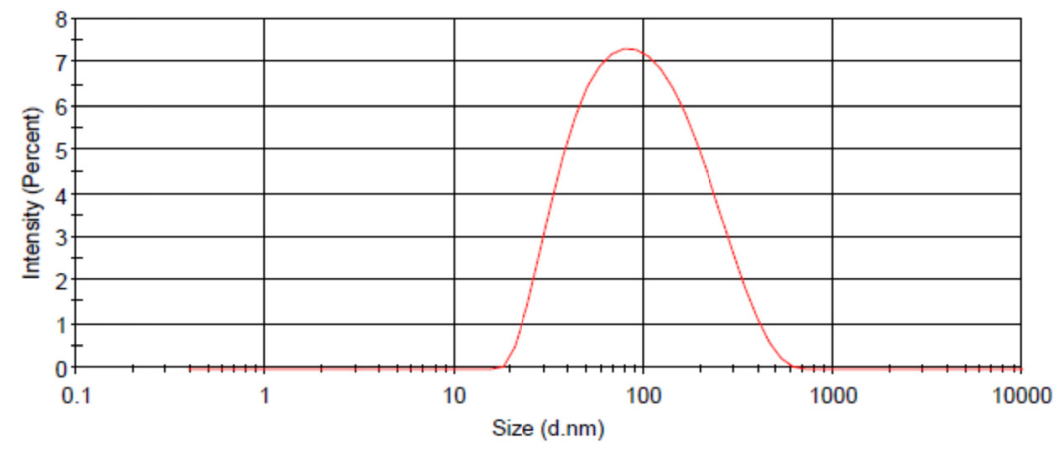

Fig. 1. The intensity based particle size analysis of the nanoemulsion

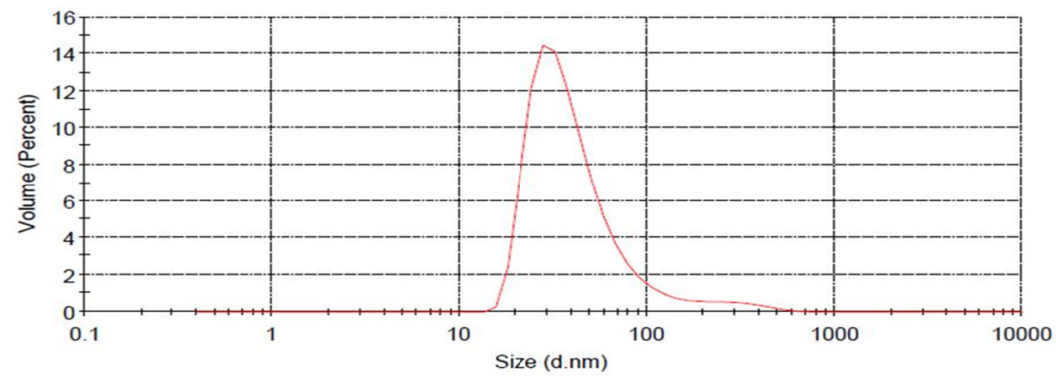

Fig. 2. The volume based particle size analysis of the nanoemulsion

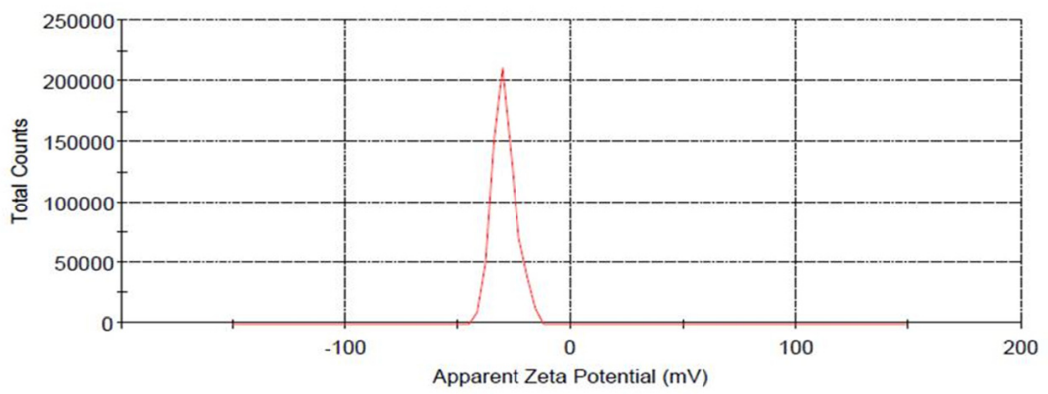

Fig. 3. The zeta potential of nanoemulsion

taining 3\% surfactant. Then, the obtained solution was poured into the vial and placed it in a water bath to reach $75^{\circ} \mathrm{C}$ and then we put the dissolved oil in the solvent into the bath to reach the desired temperature. Then, the oil as the deionized water was sterilized and then put into a centrifuge (Ultra-Turrax T25 IKA Janke and Kunkle, GmbH and $\mathrm{CO} \mathrm{KG}$ ) for 10 minutes at 1000 RMP (revolutions per minute). The sample was then kept at $4{ }^{\circ} \mathrm{C}$ for identification. Finally, homogenized solution and nanoemulsion were obtained and chemically analyzed. A semiMcFarland solution was prepared by adding $0.5 \mathrm{~mL}$ barium chloride solution $1.75 \%$ to $99.5 \% \mathrm{~mL}$ sulfuric acid $1 \%$. It is worth noting that a $0.5 \mathrm{McF}$ arland Latex Standard (used as a reference to adjust the turbidity of bacterial suspensions for susceptibility testing and other procedures that require a standardized inoculum) is comparable to a bacterial suspension of $1.5 \times 10^{8} \mathrm{CFU} / \mathrm{mL}(21,22)$.

To prepare the microbial suspension, we removed fresh and purified bacterial colonies and dissolved it in a tube containing sterile physiological serum. Then, compared with its turbidity with the standard McFarland half solution, we prepared microbial suspension equivalent to McFarland 0.5. Chlorhexidine $0.2 \%$ (positive control) and sterile serum (negative control) were used to determine microbial susceptibility. The suspensions were prepared based on 0.5 McFarland. Each plate was operated using a pour plate method. In this regard, $100 \mu \mathrm{L}$ of the standard half strain suspension of McFarland was added to the Müller Hinton Agar medium and poured into the plate to be frozen. Next, 3 wells were created per plate and the Nigella sativa nanoemulsion, positive and negative controls were added to the wells. The plates were then stored at $37^{\circ} \mathrm{C}$ for 24 hours and examined the next day in terms of the appearance of a growth inhibition zone $(12,23)$.

The diameter of the growth inhibition zone was measured with a ruler and interpreted according to the Clinical and Laboratory Standards Institute standard. The microtiter plate method (A to C, respectively) was used to determine the MIC. We used 5\% dimethylsulfoxide. We 
added 100 microliters of fresh culture medium to 9 cells per row. We assigned each row to the special bacterial species. Inside the first cell of each row, we added the following materials to obtain a final concentration of $1: 200 \mathrm{v} / \mathrm{v}(9 \%$ nanoemulsion $9 \%+$ DMSO $10 \mu \mathrm{l}+89$ of culture medium). We added 100 microliters of the first cell to the second cell and up and down 10 times. Cell no. 10 was designated for drug control (with nano-emulsion solution) and cell no. 11 for bacterial control, without nanoemulsion and bacterial cell for sterile steps control). A total of $100 \mathrm{~mL}$ microbial suspension was added to cell no. 11 and $100 \mathrm{~mL}$ sterile culture medium to cell no. 10 . In this case, we prepared the final concentrations of nanoemulsions at 1:200, 1:400, 1:800, 1:1600, 1:3200, $1: 6400$, and 1:12800 volumes (Table 1).

We added $100 \mathrm{~mL}$ of standard McFarland half microbial suspension to each row cell. Finally, we incubated it at $37^{\circ} \mathrm{C}$ for 24 to 18 hours. The lowest concentration without turbidity was considered to be MIC. To calculate the $\mathrm{MBC}$, we planted the MIC tube and the 2 preceding tubes in a plate and divided it into 3 sections. We evaluated the results after incubation. The experiments were performed in triplicate, and the mean results were reported.

After allocating the appropriate codes to the collected data, the data were entered into SPSS Version 16.0 software, and then the tests were performed experimentally using standard methods. To compare the MIC- and MBCassociated outcomes and mean diameter growth inhibition zone between $0.2 \%$ chlorhexidine mouthwash and Nigella sativa emulsion nanoemulsions, we used an independent sample $\mathrm{t}$ test and $\mathrm{P}<.05$ was considered statically significant.

\section{Results}

In this study, the effect of $0.2 \%$ CHX mouthwash and Nigella sativa emulsion nanoemulsions in different dilutions on the microbial species of Enterococcus faecalis, Streptococcus mutans, Streptococcus soberini, Streptococcus salivarius as well as Lactobacillus acidophilus were tested by the well method, the MIC method, and $\mathrm{MBC}$ assay. The values of disk diffusion and mean diame- ter of growth inhibition zone of nanoemulsion for the aforementioned species were $7,8,8,6.4$, and 8.5 , respectively, while the diameter of $\mathrm{CHX}$ inhibition zone for these bacteria was $21,20,19,30$, and $22 \mathrm{~mm}$, respectively (Table 1). Therefore, it was found that nanoemulsion of Nigella sativa had antibacterial effects on all studied microbial species. The highest and lowest growth area were specified to Lactobacillus acidophilus $(8.5 \mathrm{~mm})$ and Enterococcus faecalis $(7 \mathrm{~mm})$, respectively. The studies showed that the mean diameter of the growth inhibition zone due to nanoemulsion in different bacterial numbers was close (Table 2). However, the diameter of the CHX inhibition zone in all studied bacteria was more than $\mathrm{Ni}$ gella sativa $(p=0.665)$. Therefore, despite the nonsignificant statistical assoctation, the antibacterial affects of chlorhexidine were higher than that of nanoemulsion of Nigella sativa. However, the minimum inhibitory concentration and also the minimum lethal concentration of nanoemulsion for Enterococcus faecalis and Streptococcus mutans were similar, but higher than those for other bacteria. Therefore, it was found that the inhibitory and lethal effects of nanoemulsion were more significant in Streptococcus soberini, Streptococcus Salivarius, and Lactobacillus acidophilus bacteria. In contrast, the MIC $(p=0.042)$ and MBC $(p=0.038)$ rates of chlorhexidine for all bacteria were lower than those for the Nigella sativa nanoemulsion.

\section{Discussion}

Although the broad spectrum of antimicrobial activity of Nigella sativa has already been established, the strength of the present study is the initial assessment of the effects of different concentrations of nanoemulsion by both macro dilution and disk diffusion on the most common cariogenic bacteria (Enterococcus faecalis and Streptococcus mutans and Streptococcus sublinii and Streptococcus salubrium) and compared with chlorhexidine (17-19). Overall, it was shown that nanoemulsion of Nigella sativa significantly inhibited the growth of all studied bacteria, although its effect was less than CHX. This finding may be due to the fact that the nano drug structure at high concen-

Table 1. The compounds used to test the Minimum Inhibitory Concentration of growth

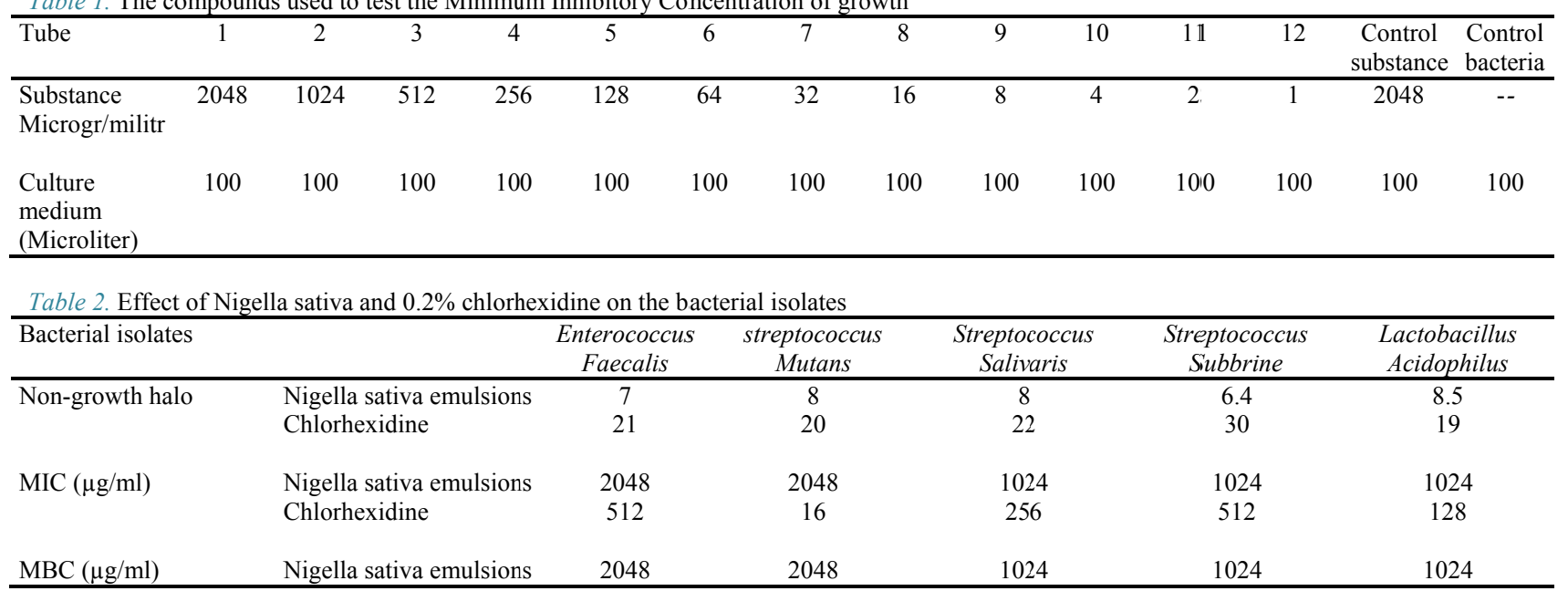


trations loses its stability, so the impossibility of producing high concentrations of the nano drug of Nigella sativa is one of the limitations of working with this plant. On the other hand, silver-based or gold-based nanoparticles may be retained if the concentration is increased, and as a result, nanoparticle concentrations can be closer to $\mathrm{CHX}$. An increase in the concentration of Nigella sativa might affect cytotoxicity, which should be considered when preparing high concentrations of this nanoparticle.

The study results by Manju et al (22), in line with the present study, showed that gold nanoparticles with Nigella sativa effectively inhibited biofilm formation of both bacteria (Vibrio harveyi and Parahemolyticus) by reducing hydrophobicity index. Therefore, it seems that the ability of Nigella sativa oil to reduce biofilm may be related to the antiplatelet and antidiabetic effects of this material. Therefore, creaming and sediment formation phenomena did not occur because the tiny droplets prevent interconnection and surface flocculation. As a result, nanoemulsions have an extremely high surface area and consequently very high permeability, making them an effective transfer system. Although the antibacterial effect of Nigella sativa and amoxicillin disc against Streptococcus mutans was similar, nanosilver showed a more significant effect against Sanguis (in contrast to the present study) (23). Due to the probable toxicity of the nanosilver, the Nigella sativa, as the less toxic substance, can be used for mouthwashing because of its antibacterial and antiseptic properties.

This study showed that the mean diameter of the inhibition zone of the nanoemulsions of all bacteria strains was close to each other. In this regard, Enterococcus faecalis and Lactobacillus acidophilus because of the lowest (7) and highest (8.5) values of the growth inhibition zone as well as high (2048) and low (1024) MIC, respectively, were the most resistant and most susceptible bacteria to Nigella sativa nanoemulsion. This outcome might be because Enterococcus faecalis is an opportunistic bacterium producing lactic acid, which is very effective in its resistance to antibiotics. Therefore, its resistance (as Streptococcus group D) to nanoemulsion is predictable (24). Also, the present study showed that the antimicrobial effect of Nigella sativa nanoemulsion on growth inhibition of Enterococcus faecalis and Streptococcus mutans was more negligible than other bacteria. In contrast, its inhibitory effects on Lactobacillus acidophilus, Streptococcus salivarius, and Soberini were similar and may be because of the structural differences of the bacteria. In this regard, an in vitro study by Kouidhi et al (25), similar to the present study, showed that the addition of thymoquinone in Nigella sativa has selective antimicrobial effects. Simultaneously, it is effective against gram-positive bacteria, but gram-negative bacteria are resistant to it, and its synergistic effects enhance antimicrobial drugs. Besides, it has been shown that Nigella sativa oil at appropriate concentrations can inhibit the growth of methicillin-resistant Staphylococcus bacteria. Therefore, synergistic use of Nigella sativa with other antimicrobial drugs can be suggested. The significant antibacterial effect of Nigella sativa oil against a variety of bacteria might appear to be due to their different structure and mechanisms. In this way, the physiology of the cell envelope of gram-negative bacteria and having an outer layer on the outer membrane varied in sensitivity and consequently the absorption identification. Thus, the outer membrane reduces access to the Nigella sativa molecules (due to their molecular size) to a definite expansion and results in the killing of bacteria but accelerates the discovery of organelles. Also, Arici et al (26) studied the antibacterial effects of 5 species of Turkish Nigella sativa on 24 different bacterial strains concurrent with the present study, which showed that lactic acidproducing bacteria were more resistant to Nigella sativa oil than other bacteria. Given that all the bacteria studied, including Streptococcus, Enterococci, and Lactobacillus, were lactic acid producing bacteria (LAB lactic acid bacteria), the weaker antibacterial power of the Nigella sativa nanoemulsion compared with $\mathrm{CHX}$ was attributed to these bacteria. Moreover, it should be noted that the results of other studies with Nigella sativa species from other geographical areas cannot be generalized to other studies because of the structural differences of native herbal remedies in different regions $(25,26)$. In recent years, because of topical (skin allergy) and systemic (nephrotoxicity and hepatotoxic) side effects of Nigella sativa seed, most studies have investigated the effect of active part of Nigella sativa (Thymoquinone). In this regard, Kokoska et al study (27), in line with the present study, showed that the Nigella sativa extracted by the broth microdelution method (BMDM) could inhibit the growth of pathogenic oral bacteria, especially Streptococcus mutans and subrinus; however, the difference in statistical values may be related to the different drug structure studied (28). The study by Harzallah et al (29), in line with the present study of the antimicrobial effects of Nigella sativa seed (Tunisia species) extracted by BMDM, showed that Nigella sativa oil had a dose-dependent antibacterial effect. In contrast, pure thymoquinone was effective against all studied strains, especially Sturgeon, and its growth inhibitory effects on human epithelial cells were strong. Mohammed (30) who investigated the effect of 2 different types of black Nigella sativa extract on 2 cariogenicic bacteria (Streptococcus mutans and methis) stated that methanolic extract of Nigella sativa seed (with $6.3 \mathrm{~mm}$ area inhibitory value against Streptococcus mutan) was more effective than ethanolic extract $(12.7 \mathrm{~mm}$ diameter $)$. However, in this study, the effect of Nigella sativa nano-drug was compared with CHX in a various common cariogenicic bacteria. Sufya et al (31) study showed that the Nigella sativa oil extract of Libya had a significant effect on Escherichia coli and Streptococcus aureus but had a greater effect on Streptococcus aureus and a more significant effect on Streptococcus aureus. However, unlike the 2 above studies, the effect of different concentrations of nano drug on bacterial species was investigated in the present study, as toxicity evaluations about different concentrations are necessary for therapeutic assessments and oral flora contain various bacteria $(32,33)$.

In the in vitro study of Ugur AR, et al, titled as "Antibacterial Activity of Nigella sativa Oil Against grampositive and gram-negative bacteria, consistent results 
were shown with other researches in terms of grampositive bacteria. Ugur study showed that Nigella sativa oil compared with the control group had no cytotoxic effect on the proliferation of the gingival fibroblasts. Nigella sativa was very active against Methicillin-Resistant CoNS and Methicillin-Resistant Staphylococcus aureus, which had no in vitro cytotoxicity at relevant concentrations up to $1 \mu \mathrm{g} / \mathrm{mL}$. It seems that antimicrobial activities of a specific plant may differ depending on geographical location where it has been cultivated (34).

The effect of Nigella sativa on caries can be since, in addition to its antimicrobial effect, Nigella sativa seed oil inhibits the adhesion of streptococcus mutans to the tooth surface. The antimicrobial mechanism of Nigella sativa is not yet well understood. However, it may be related to active compounds, particularly thymoquinone and melanin, and the widespread activity of these components can also be due to the impact on critical processes of microorganisms.

\section{The strengths of study}

Nano drugs have recently been developed and introduced because of their benefits (more extended durability and targeted tissue distribution, and reduced side effects) as a novel production method that has been of interest to researchers in various clinical medicine disciplines; and in this study, for the first time, the antibacterial effects of nanoemulsion of Nigella sativa were investigated by broth macro dilution and disk diffusion methods on the most common cariogenic and compared with standard control drug (Chlorhexidine). Since in the present study, as the first study, we were faced with limitations in selecting bacterial species, only the common oral microbes, mostly $\mathrm{LAB}$, were investigated. In the case of molecular, cellular studies on the mechanism of its action, the use of this herb in toothpaste and other oral hygiene products is recommended. Finally, it is recommended that other researchers investigate the inhibitory effects of different concentrations of Nigella sativa and other nanocarriers with different sizes on-resistance of bacterial species.

\section{Conclusion}

Overall, the present study showed that Nigella sativa nanoemulsions were effective on common cariogenic pathogens, as Enterococcus faecalis and Lactobacillus faecalis were more resistant and more sensitive species to this nanoemulsion respectively, however, the antimicrobial effects of Nigella sativa nanoemulsions were weaker than the standard drug of chlorhexidine.

\section{Acknowledgement}

The authors would like to thank the Research Council of Zanjan University of Medical Sciences and all the patients who kindly participated in this study.

\section{Conflict of Interests}

The authors declare that they have no competing interests.
1. Lima SLA, Santana CCP, Paschoal MAB, Paiva SM, Ferreira MC. Impact of untreated dental caries on the quality of life of Brazilian children: population-based study. Int J Paediatr Dent. 2018;28(4):390399

2. Van de Rijt LJM, Stoop CC, Weijenberg RAF, de Vries R, Feast AR, Sampson EL, et al. The Influence of Oral Health Factors on the Quality of Life in Older People: A Systematic Review. Gerontologist. 2020;60(5):e378-e394.

3. Cummins D. Dental caries: a disease which remains a public health concern in the 21st century--the exploration of a breakthrough technology for caries prevention. J Clin Dent. 2013;24:1-14.

4. Struzycka I. The oral microbiome in dental caries. Pol J Microbiol. 2014;63(2):127-35.

5. Aas JA, Griffen AL, Dardis SR, Lee AM, Olsen I, Dewhirst FE, et al. Bacteria of dental caries in primary and permanent teeth in children and young adults. J Clin Microbiol. 2008;46(4):1407-17.

6. Adyatmaka AU, Sutopo P, Carlsson D, Bratthall G, Pakhomov A. School-Based Primary Preventive Programme for Children: Affordable Toothpaste as a Component in Primary Oral Health CareExperiences from a Field Trial in Kalimantan Barat, Indonesia. Geneva: World Health Organization. 1998

7. Addy M. Plaque control as a scientific basis for the prevention of dental caries. J R Soc Med. 1986;79 (Suppl 14):6-10.

8. Ruiz-Linares M, Ferrer-Luque CMM, Arias-Moliz T, de Castro P, Aguado B, Baca P. Antimicrobial activity of alexidine, chlorhexidine and cetrimide against Streptococcus mutans biofilm. Ann Clin Microbiol Antimicrob. 2014;20;13:41.

9. Barrios R, Ferrer-Luque CM, Arias-Moliz MT, Ruiz-Linares M, Bravo M, Baca P. Antimicrobiall substantivity of alexidine and chlorhexidine in dentin. J Endod. 2013;39(11):1413-5.

10.Cieplik F, Jakubovics NS, Buchalla W, Maisch T, Hellwig E, AlAhmad A. Resistance Toward Chlorhexidine in Oral Bacteria - Is There Cause for Concern? Front Microbiol. 2019;22(10):587.

11. Hanafy MS, Hatem ME. Studies on the antimicrobial activity of Nigella sativa seed (black cumin). J Ethnopharmacol. 1991;34(23):275-8.

12. Bakathir HA, Abbas NA. Detection of the antibacterial effect of Nigella sativa ground seeds with water. Afr J Tradit Complement Altern Med. 2011;8(2):159-64

13. Hamdan A, Haji Idrus R, Mokhtar MH. Effects of Nigella Sativa on Type-2 Diabetes Mellitus: A Systematic Review. Int J Environ Res Public Health. 2019,5;16(24).

14. Hussain SA, Mohammed Ameen HA, Mohammed MO, Ahmed KM, Hama-Gareb Ali R, Safar BM, et al. 2019. Nigella sativa Oil Mouth Rinse Improves Chemotherapy-Induced Oral Mucositis in Patients with Acute Myeloid Leukemia. Biomed Res Int. 2019;30:3619357.

15. Ahmad A, Husain A, Mujeeb M, Khan SA, Najmi AK, Siddique NA, et al. A review on therapeutic potential of Nigella sativa: A miracle herb. Asian Pac J Trop Biomed. 2013;3(5):337-52.

16. Gholamnezhad Z, Havakhah S, Boskabady MH. Preclinical and clinical effects of Nigella sativa and its constituent, thymoquinone: A review. J Ethnopharmacol. 2016;22(190):372-86.

17. Amin B, Hosseinzadeh H. Black Cumin (Nigella sativa) and Its Active Constituent, Thymoquinone: An Overview on the Analgesic and Anti-inflammatory Effects. Planta Med. 2016;82(1-2):8-16.

18. Besra M, Kumar V. In vitro investigation of antimicrobial activities of ethnomedicinal plants against dental caries pathogens. 3 Biotech. 2018;8(5):257

19. Al-Attass SA, Zahran FM, Turkistany SA. Nigella sativa and its active constituent thymoquinone in oral health. Saudi Med J. 2016;37(3):235-44

20. Kiari FZ, Meddah B, Tir Touil Meddah A. In vitro study on the activity of essential oil and methanolic extract from Algerian Nigella sativa L. Seeds on the growth kinetics of micro-organisms isolated from the buccal cavities of periodontal patients. Saudi Dent J. 2018;30(4):312-323.

21. Amooaghaie R, Saeri MR, Azizi M. Synthesis, characterization and biocompatibility of silver nanoparticles synthesized from Nigella sativa leaf extract in comparison with chemical silver nanoparticles. Ecotoxicol Environ Saf. 2015;120:400-8.

22. Manju S, Malaikozhundan B, Vijayakumar S, Shanthi S, Jaishabanu A, Ekambaram P, et al. Antibacterial, antibiofilm and cytotoxic effects of Nigella sativa essential oil coated gold nanoparticles. Microb Pathog. 2016;91:129-35.

\section{References}


23. Abd-Awn B, Al-Dhaher Z, Al-Dafaai R. The effect of black seed oil extracts on mutans streptococci in comparison to chlorhexidine gluconate (in vitro). J Baghdad Coll Dent. 2012;24:126-131.

24. Forouzanfar F, Fazly Bazzaz BS, Hosseinzadeh H. Black cumin (Nigella sativa) and its constituent (thymoquinone): a review on antimicrobial effects. Iran J Basic Med Sci. 2014;17:929-938.

25. Chaieb K, Kouidhi B, Jrah H, Mahdouani K, Bakhrouf A. Antibacterial activity of Thymoquinone, an active principle of Nigella sativa and its potency to prevent bacterial biofilm formation. BMC Compl Altern Med. 2011;11:29.

26. Sapmaz E, Sapmaz HI, Vardi N, Tas U, Sarsilmaz M, Toplu Y, et al. Harmful effects of formaldehyde and possible protective effect of Nigella sativa on the trachea of rats. Niger J Clin Pract. 2017;20(5):523-529.

27. Novy P, Kloucek P, Rondevaldova J, Havlik J, Kourimska L, Kokoska L. Thymoquinone vapor significantly affects the results of Staphylococcus aureus sensitivity tests using the standard broth microdilution method. Fitoterapia. 2014;94:102-7.

28. Shabestari SB, Shirinbak I, Agha-Hosseini F. Maxillary metastasis of a medullary thyroid carcinoma in a 21-yearold woman 7 years after thyroidectomy. J Oral Maxillofac Surg. 2012;70:1495-9

29. Harzallah HJ, Grayaa R, Kharoubi W, Maaloul A, Hammami M, Mahjoub T. Thymoquinone, the Nigella sativa bioactive compound, prevents circulatory oxidative stress caused by 1,2-dimethylhydrazine in erythrocyte during colon postinitiation carcinogenesis. Oxid Med Cell Longev. 2012;2012:854065.

30. Mohammed NK, Abd Manap MY, Tan CP, Muhialdin BJ, Alhelli AM, Meor Hussin AS. The Effects of Different Extraction Methods on Antioxidant Properties, Chemical Composition, and Thermal Behavior of Black Seed (Nigella sativa L) Oil. Evid Based Complement Alternat Med. 2016;2016:6273817.

31. Sufya NM, Walli RR, Wali FM, Alareiba MS, Doro BM. Studies of the antimicrobial activity of Black Seed Oil from Nigella Sativaon Staphylococcus aureus and Escherichia coli. J Libyan Med Res. 2014;8(1):59-68.

32. Shabestari SB, Shirinbak I, Azadarmaki R. Cancer Genetics and Psychotherapy: A comprehensive look at oromaxillofacial and laryngopharyngeal cancers. Cancer Genet Psychother. 2017;531-87.

33. Schackert HK, Agha-Hosseini F, Gorgens H, Jatzwauk M, von Kannen S, Noack B, et al. Complete homozygous deletion of CTSC in an Iranian family with Papillon-Lefevre syndrome. Int J Dermatol. 2014;53:885-7

34. Ugur AR, Dagi HT, Ozturk B, Tekin G, Findik D. Assessment of in vitro antibacterial activity and cytotoxicity effect of Nigella sativa oil. Phcog Mag. 2016;12(Suppl S4):471-4. 